\title{
Capping of Silybin with $\beta$-Cyclodextrin Influences its Binding with Bovine Serum Albumin: A Study by Fluorescence Spectroscopy and Molecular Modeling
}

\author{
Sudha Natesan, Chandrasekaran Sowrirajan, Premnath Dhanaraj, ${ }^{\dagger}$ and Israel V. M. V. Enoch ${ }^{*}$ \\ Department of Chemistry, School of Science and Humanities, Karunya University, Coimbatore 641 114, Tamil Nadu, India \\ ${ }^{*}$ E-mail: drisraelenoch@gmail.com \\ ${ }^{\dagger}$ Department of Bio-Informatics, School of Biotechnology and Health Sciences, Karunya University, \\ Coimbatore 641 114, Tamil Nadu, India \\ Received February 7, 2014, Accepted March 21, 2014
}

\begin{abstract}
The association of silybin with $\beta$-cyclodextrin and its influence on silybin's binding with bovine serum albumin are reported. The stoichiometry, binding constant, and the structure of silybin- $\beta$-cyclodextrin inclusion complex are reported. The titrations of silybin with bovine serum albumin in the absence and presence of $\beta$ cyclodextrin are carried out and the differences in binding strengths are discussed. Molecular modeling is used to optimize the sites and mode of binding of silybin with bovine serum albumin. Förster resonance energy transfer is calculated and the proximity of interacting molecules is reported in the presence and absence of $\beta$ cyclodextrin.
\end{abstract}

Key Words : Silybin, $\beta$-Cyclodextrin, Bovine serum albumin, FRET, 2D ROESY

\section{Introduction}

Binding of drugs with proteins plays a potential role in pharmacokinetic ${ }^{1-3}$ and pharmacodynamic properties of drugs. ${ }^{4}$ The binding equilibrium and the offset rate may influence the efficacy and the distribution of the bound small molecule. ${ }^{5}$ Studies are carried out to evaluate the effect of concentration, temperature, $\mathrm{pH}$ and ionic strength on highly albumin-bound drugs and bovine serum albumin (BSA) interaction to explore protein-binding characteristics of drugs. ${ }^{6,7}$ Serum albumins are the major soluble protein constituents of the circulatory system; they serve as transport proteins for numerous endogenous and exogenous compounds. The exogenous substances have a high affinity for proteins. This interaction between protein and drug molecules results in a stable protein-drug complex formed. BSA is a globular protein and has a compact ellipsoid structure. The stability of this structure originates mainly from hydrophobic interactions. $^{8-10}$

Silymarin is the biological extract of the dried seeds of the herb Silybum marianum and silybin is the major active constituent of silymarin. ${ }^{11}$ Silybin (SIL) has anti-inflammatory, anti-oxidative and anti-carcinogenic properties. ${ }^{12-14}$ It is used in the treatment of a variety of diseases related to liver, prostate gland, lungs, kidneys, pancreas, and skin. ${ }^{15-17}$ The structure-antioxidant activity relationship of flavonoids has been extensively studied. ${ }^{18,19}$ However, the mechanism of action of silybin and its derivatives as radical scavengers and antioxidants is rarely studied ${ }^{20}$ and it remains unclear.

Cyclodextrins (CDs) have been extensively studied and recognized as pharmaceutical excipients. Their molecular structures render them interesting molecular host systems with a unique property like hydrophilic exterior and a hydrophobic central cavity. ${ }^{21}$ This enables them to form non- covalent inclusion complexes by encapsulation of small molecules into their hydrophobic cavities. These non-covalent inclusion complexes have a number of physicochemical advantages over un-manipulated drugs. CDs are insoluble in most organic solvents and are soluble in water. The encapsulation protects the drug molecule against attack by various reactive molecules and reduces the rate of hydrolysis, oxidation, rearrangement, racemization and enzymatic decomposition. ${ }^{22}$ Although CDs are established drug carriers reports on the binding of drugs with serum albumins in CD-loaded form are scarce in the literature. ${ }^{23}$ Studies must be carried out on binding of small molecules in CD-bound form with proteins in order to understand the ability of CDs in transporting drugs and to know about the percentage availability of drugs at the targeted site. In this paper we discuss the binding of a derivative of silybin in free form and in $\beta$ cyclodextrin $(\beta-C D)$ complexed form.

\section{Experimental Section}

Materials and Methods. Silybin was purchased from Sigma-Aldrich, Bangalore, India. $\beta$-Cyclodextrin was obtained from Hi Media and used as received. Effect of $\mathrm{pH}$ studies were done by the various $\mathrm{pH}$ solutions adjusted with 0.1 mmol of phosphoric acid and $0.1 \mathrm{mmol}$ of sodium hydroxide solutions. The measurement of $\mathrm{H}_{0}$ and $\mathrm{pH}$ value below the pH 2 from a modified Hammett's acidity scale. ${ }^{26}$ Crystalline bovine serum albumin and HEPES buffer were purchased from Hi Media. All the solvents used from Merck were of spectral grade which were used as received.

Preparation of Test Solutions. Test solutions were prepared by appropriate dilution of a stock solution of SIL. The stock solution of SIL was made in methanol due to its less solubility of in water. The test solutions were having the 
concentration of methanol as $1 \%$. HEPES buffer $(0.1 \mathrm{mmol})$ was used to prepare stock solution of BSA of concentration $3.0 \times 10^{-5} \mathrm{mmol}$. Doubly distilled water was used throughout the experiments. All experiments were carried out at ambient temperature of $25 \pm 2{ }^{\circ} \mathrm{C}$. The test solutions were homogeneous after the addition of all additives. The absorption and the fluorescence spectra were recorded against appropriate blank solutions.

Preparation of SIL- $\boldsymbol{\beta}$-CD Solid Complex. In a $50 \mathrm{~mL}$ beaker, SIL $\left(0.25 \mathrm{~g}, 5.18 \times 10^{-4} \mathrm{mmol}\right)$ was dissolved in $5 \mathrm{~mL}$ of methanol. $5 \mathrm{~mL}$ of aqueous $\beta$-CD $\left(0.59 \mathrm{~g}, 5.18 \times 10^{-4}\right.$ $\mathrm{mmol}$ ) was prepared in another $50 \mathrm{~mL}$ beaker separately. A solution of SIL was added slowly to the solution of $\beta-C D$ at room temperature and sonicated in an Ultra-sonicator for 30 minutes to get a homogenous solution. Then the solution was warmed to $50{ }^{\circ} \mathrm{C}$ for $10-15$ minutes and kept at room temperature for two days. The solid precipitated was analyzed with spectroscopic techniques.

Instrumentation. A double beam UV-Visible spectrophotometer, Jasco V-630 was used to study absorption measurements using $1 \mathrm{~cm}$ path length cells. Jasco FP-750 spectrofluorimeter equipped with a $120 \mathrm{~W}$ Xenon lamp for excitation served the measurement of fluorescence. Both the excitation and the emission band widths were set up at 2.5 $\mathrm{nm}$. Time-resolved fluorescence measurements were done on a time-correlated single photon counting HORIBA spectrofluorimeter. An ultra-sonicator, PCI 9L 250H, India was used for sonication. Various $\mathrm{pH}$ solutions were adjusted using Elico LI $120 \mathrm{pH}$ meter, India. ${ }^{1} \mathrm{H}$ NMR and 2D ROESY spectrum were recorded on a Bruker AV III instrument operating at $500 \mathrm{MHz}$ with and an internal standard was Tetramethylsilane (TMS) used. The chemical shift values were obtained downfield from TMS in part per million (ppm). The mixing time for ROSEY spectra was $200 \mathrm{~ms}$ under the spin lock condition. DMSO- $d_{6}$ was used in the ${ }^{1} \mathrm{H}$ NMR and the 2D ROESY NMR experiment for the analysis of SIL and SIL $-\beta-C D$ solid complex respectively.

\section{Results and Discussion}

Complexation of SIL with $\boldsymbol{\beta}$-CD. The absorption spectra of SIL in water and at different concentrations of $\beta$-CD are shown in Figure 1(a). The absorption and the fluorescence spectral data of SIL in the absence and the presence of $\beta-C D$ are compiled in Table 1. SIL shows two absorption maxima viz. $285 \mathrm{~nm}$ and $326 \mathrm{~nm}$ in the absence of $\beta$-CD. On addition of $\beta$-CD the absorptions of both the bands shift by about $1 \mathrm{~nm}$ towards shorter wavelengths. More significantly, the absorption maximum at $285 \mathrm{~nm}$ shows a hyperchromic shift on the addition of $\beta$-CD whereas the $326 \mathrm{~nm}$ band shows a general hypsochromic shift. These spectral changes are ascribed to the formation of a complex between SIL and $\beta$ $\mathrm{CD}$ as represented by Eq. (1)

$$
\mathrm{SIL}+\beta-\mathrm{CD} \rightleftarrows \mathrm{SIL}-\beta-\mathrm{CD}
$$

The occurrence of structural modification induced in the
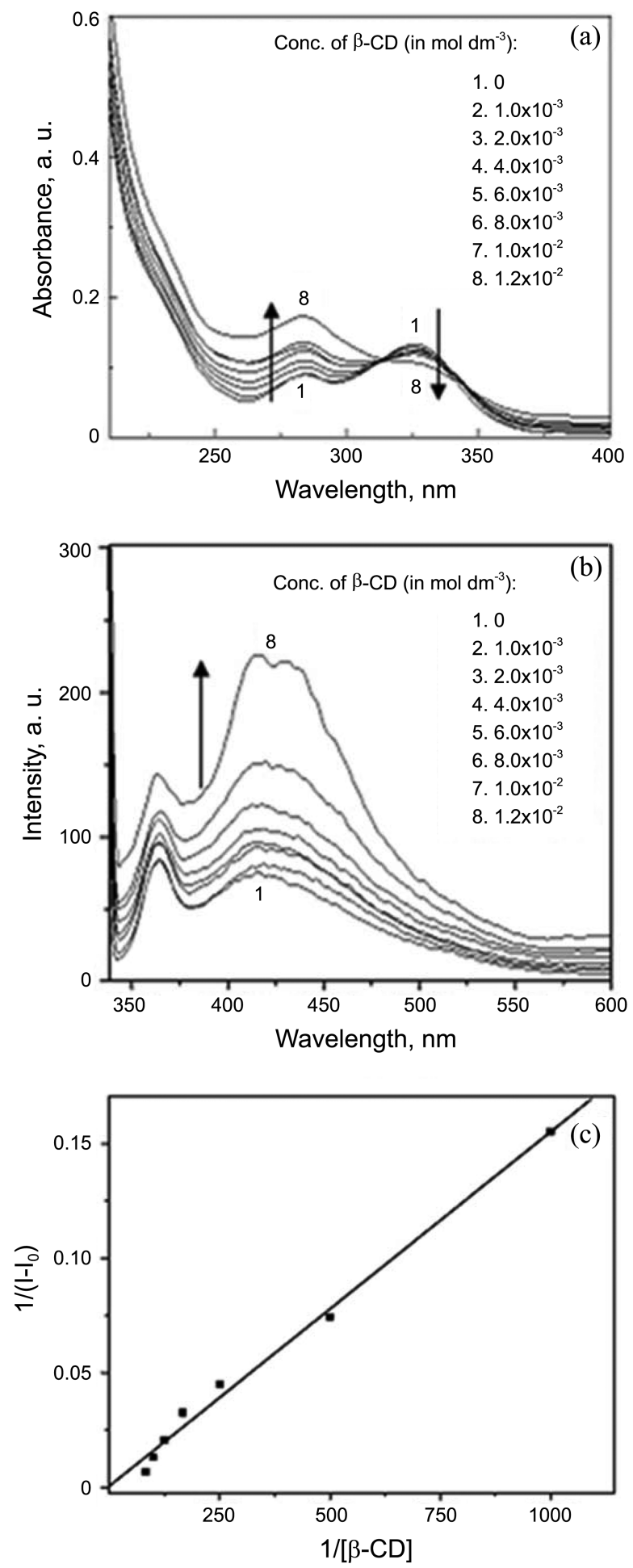

Figure 1. (a) Absorption spectra of SIL with various concentrations of $\beta-C D$. (b) Fluorescence spectra of SIL with various concentrations of $\beta-\mathrm{CD}$. (c) Benesi-Hildebrand plot for $1: 1$ complex of SIL- $\beta$-CD complex.

system by the interaction between SIL and $\beta$-CD molecules leads to free movement of electrons into different energy levels. More UV light is absorbed and the electronic transition probability of SIL electrons increases. Thus the magnitude of absorbance increases. 
Table 1. Absorption and fluorescence spectral data for the binding titration of SIL with $\beta$-CD

\begin{tabular}{cccc}
\hline $\begin{array}{c}\text { Concentration of } \\
\beta-\mathrm{CD}, \mathrm{mol} \mathrm{dm}^{-3}\end{array}$ & $\begin{array}{c}\text { Absorption } \\
\text { maxima, nm }\end{array}$ & $\begin{array}{c}\text { Absorbance, } \\
\text { a. u. }\end{array}$ & $\begin{array}{c}\text { Fluorescence } \\
\text { maxima, nm }\end{array}$ \\
\hline \multirow{2}{*}{0} & 285.0 & 0.090 & 415.0 \\
& 326.0 & 0.133 & \\
$1.0 \times 10^{-3}$ & 285.0 & 0.089 & 419.0 \\
& 326.0 & 0.128 & \\
$2.0 \times 10^{-3}$ & 285.0 & 0.100 & 426.5 \\
& 326.0 & 0.128 & \\
$4.0 \times 10^{-3}$ & 284.0 & 0.109 & 423.0 \\
& 326.0 & 0.123 & \\
$6.0 \times 10^{-3}$ & 284.0 & 0.123 & 423.5 \\
& 325.0 & 0.122 & \\
$8.0 \times 10^{-3}$ & 284.0 & 0.128 & 430.0 \\
& 325.0 & 0.119 & \\
$1.0 \times 10^{-2}$ & 284.0 & 0.135 & 439.0 \\
& 325.0 & 0.119 & \\
$1.2 \times 10^{-2}$ & 283.0 & 0.172 & 447.5 \\
& 325.0 & 0.107 & \\
\hline
\end{tabular}

Note: Excitation wavelength: $326 \mathrm{~nm}$

The changes in the fluorescence intensity and the spectra of SIL where used to evaluate the binding constant as the fluorescence spectral changes are more pronounced than those of absorption spectra. SIL was excited at the isosbestic point for the complex formation $318 \mathrm{~nm}$, in the absence and presence of various concentrations of $\beta$-CD $\left(0\right.$ to $1.2 \times 10^{-2}$ mol dm${ }^{-3}$ ).

There are two fluorescence maxima observed for SIL, one at $364 \mathrm{~nm}$ and the other at $415 \mathrm{~nm}$ [Fig. 1(b)]. The shorter wavelength band $(364 \mathrm{~nm})$ is not much altered on the addition of $\beta-\mathrm{CD}$. There is increase in the intensity of fluorescence at this wavelength. However, the longer wavelength band at $415 \mathrm{~nm}$ (in water) shows a large red shift to 447.5 $\mathrm{nm}$ with the highest concentration of $\beta$-CD $\left(1.2 \times 10^{-2} \mathrm{~mol}\right.$ $\mathrm{dm}^{-3}$ ). The large red shift indicates the presence of strong binding between SIL and $\beta-C D$ and suggests a binding force more influencing than a simple shift of SIL to the non-polar cavity of $\beta$-CD from the surrounding polar shell of water molecules. This band also shows a fluorescence enhancement. It is well established that a partial or total encapsulation of a fluorescent molecule by the CD cavity. These evidences demonstrate that inclusion complex formation occurs between SIL and $\beta$-CD. On encapsulation SIL experiences an environment chemically different from aqueous solution (restriction in geometry and reduction of polarity). This significantly influences the dynamics of the absorption and fluorescence processes of SIL.

The fluorescence enhancement i.e., the increase in the intensity of fluorescence is used in the estimation of the binding constant. The fluorescence intensity changes are more pronounced than the absorbance changes in UV-visible spectrum. Hence fluorescence spectral data are chosen to estimate the stoichiometry and the binding constant of the SIL- $\beta$-CD complex using the modified Benesi-Hildebrand equation $^{24,25}$ (Eq. 2) which relates the fluorescence changes to the binding constant $(\mathrm{K})$ for the complex as

$$
\frac{1}{I-I_{0}}=\frac{1}{I^{\prime}-I_{0}}+\frac{1}{I^{\prime}-I_{0}} \frac{1}{k[\beta-\mathrm{CD}]}
$$

where $I_{0}$ and $I$ are the respective fluorescence intensities at $420 \mathrm{~nm}$ in the absence and presence of different concentration of $\beta-\mathrm{CD}, I^{\prime}$ is the highest concentration of $\beta-\mathrm{CD}, k$ is the binding constant. Figure 1(c) shows the Benesi-Hildebrand plot for the complex formation resulting in a binding constant of $42.88 \mathrm{~mol}^{-1} \mathrm{dm}^{3}$.

Effect of $\mathrm{pH}$ on the Binding of SIL with $\beta$-CD. The absorbance of a compound may increase or decrease with change of $\mathrm{pH}$ and a changed spectrum results on protonation of a particular functional group in the compound. The $\mathrm{p} K_{\mathrm{a}}$ of the group can be evaluated from the absorbance data using the following relation:

$$
\begin{aligned}
\mathrm{pH} & =\mathrm{p} K_{\mathrm{a}}-\frac{[\text { protonated form }]}{[\text { deprotonated form }]} \\
& =\mathrm{p} K_{\mathrm{a}}-\log \frac{\left[\mathrm{A}_{\mathrm{j}}-\mathrm{A}_{\max }\right]}{\left[\mathrm{A}_{\min }-\mathrm{A}_{\mathrm{j}}\right]}
\end{aligned}
$$

if the peak intensity at wavelength $\lambda$ decreases, and

$$
=\mathrm{p} K_{\mathrm{a}}-\log \frac{\left[\mathrm{A}_{\min }-\mathrm{A}_{\mathrm{j}}\right]}{\left[\mathrm{A}_{\mathrm{j}}-\mathrm{A}_{\max }\right]}
$$

if the peak intensity at wavelength $\lambda$ increases. The total concentration of acid (or conjugate acid) is proportional to $A_{\min }$ and the total concentration of conjugate base is proportional to $A_{\max } A_{j}$ is the absorbance at wavelength $\lambda$ of the $j^{\text {th }}$ solution between the two extremes. In equation (5), $A_{\max }$ and $A_{\text {min }}$ play just the reverse role.

The ease of access to the guest molecule complexed in $\beta$ $\mathrm{CD}$ can be understood by the neutral-protonation equilibrium of the guest molecule in the presence and the absence of $\beta$ $\mathrm{CD}$. When the $\mathrm{pH}$ is decreased from 7 , there is decrease of absorbance of SIL at the longer wavelength band $(321 \mathrm{~nm})$ continually down to high acid concentration $\mathrm{H}_{0},-1.38$ [Fig. 2(a)]. The absorption of the shorter wavelength band increases correspondingly. There is an isosbestic point suggesting equilibrium between a neutral and cationic form of SIL as there are oxygen atoms present in the molecule which are possible deprotonated. However, the actual site of protonation could not be found out. The ground state $\mathrm{p} K_{\mathrm{a}}$ for the neutral-cation equilibrium of SIL is calculated using the relations described above as 5.24. This clear isosbestic point is not observed for the same equilibrium of SIL in the presence of $\beta$-CD [Fig. 2(b)]. Hence it is an indication that the prototropic equilibrium is affected by the encapsulation of $\beta-C D$ as the protected environment in $\beta-C D$ offers a restriction for the acid strength to interfere with the hydroxyls of the SIL molecule freely.

The ${ }^{1} \mathrm{H}$ NMR spectrum of SIL is given in Figure 3. The hydroxyl groups of benzopyran moiety in the positions 3,5 and 7 are resonated as singlets in the chemical shift of 5.77, 12.0 and $10.85 \mathrm{ppm}$ respectively. The signals due to $\mathrm{CH}$ 

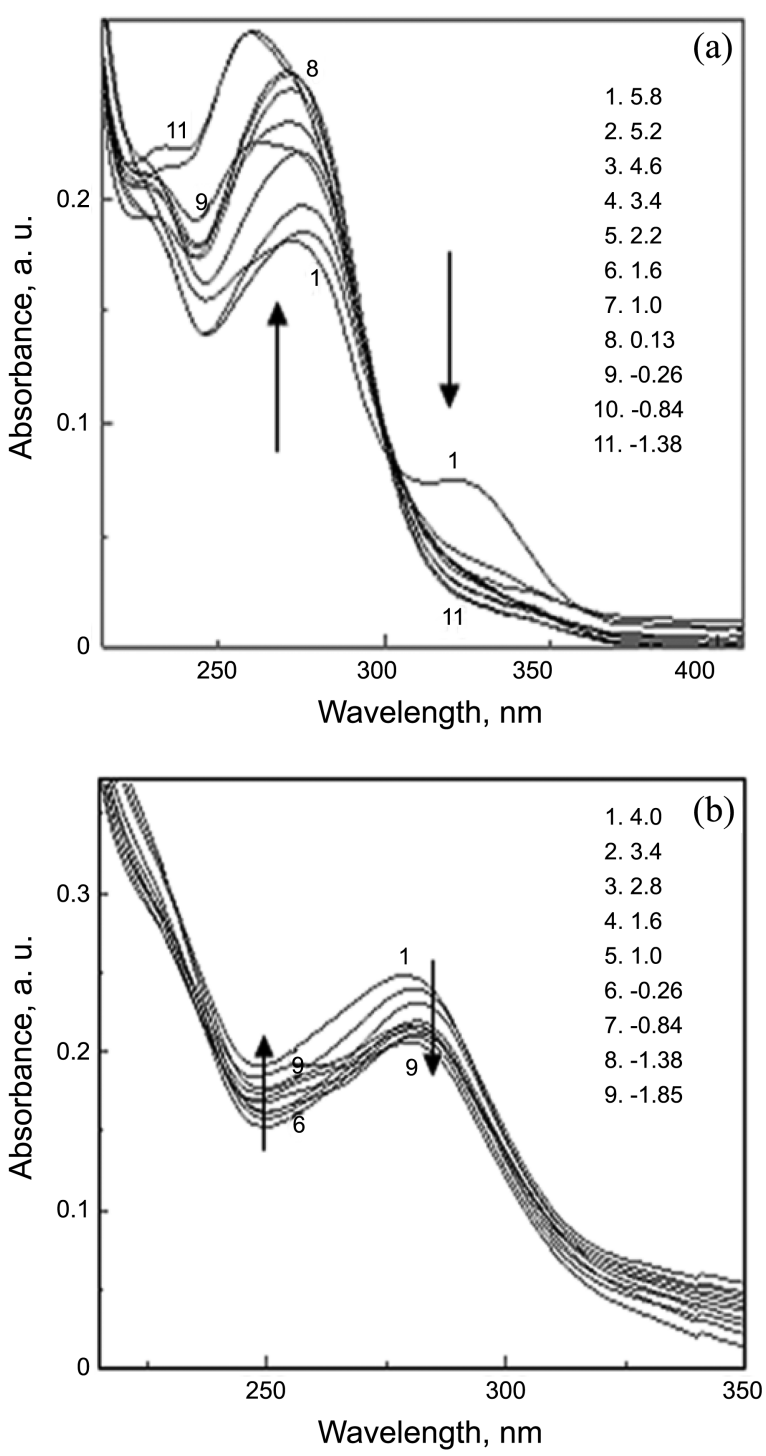

Figure 2. (a) Effect of $\mathrm{pH}$ on the absorption spectrum of SIL in water (b) Effect of $\mathrm{pH}$ on the absorption spectrum of SIL in $\beta$-CD.

protons present in the positions 2 and 3 of the dihydrobenzopyran are observed at $5.01 \mathrm{ppm}$ as doublet and a multiplet centered at $4.62 \mathrm{ppm}$ respectively. Similarly, the protons of hydrogen present in the benzodioxin ring, at positions $2^{\prime}$ and $3^{\prime}$, appear at $4.92 \mathrm{ppm}$ as doublet and $4.17 \mathrm{ppm}$ as a multiplet for respectively. The singlet at $1.9 \mathrm{ppm}$ is assigned to the hydroxyl group attached to the $\mathrm{CH}_{2}$ in the position $3^{\prime}$. The protons of $\mathrm{CH}_{2}$ in the same position resonate at $3.79 \mathrm{ppm}$. The methoxy protons are at $3.77 \mathrm{ppm}$, it is very close to methylene protons. The hydroxyl proton of 4 " is found as a triplet in the range of 9.11 to $9.29 \mathrm{ppm}$. The aromatic proton signals of SIL are observed in the range of 6.64 to $7.10 \mathrm{ppm}$.

Figure 4 shows the 2D ROESY NMR spectra of SIL- $\beta$ $\mathrm{CD}$ complex. The spectrum shows off-diagonal peaks due to the correlation of the signals of SIL to the $\beta$-CD. The hydroxyl proton signal of SIL, at position 4", cross-correlates with the signal due to $\mathrm{H} 3$ proton which is lined in the middle of $\beta$-CD cavity (denoted as 1 in the spectrum). The methoxy protons (position $3^{\prime \prime}$ ) are cross correlate to the primary

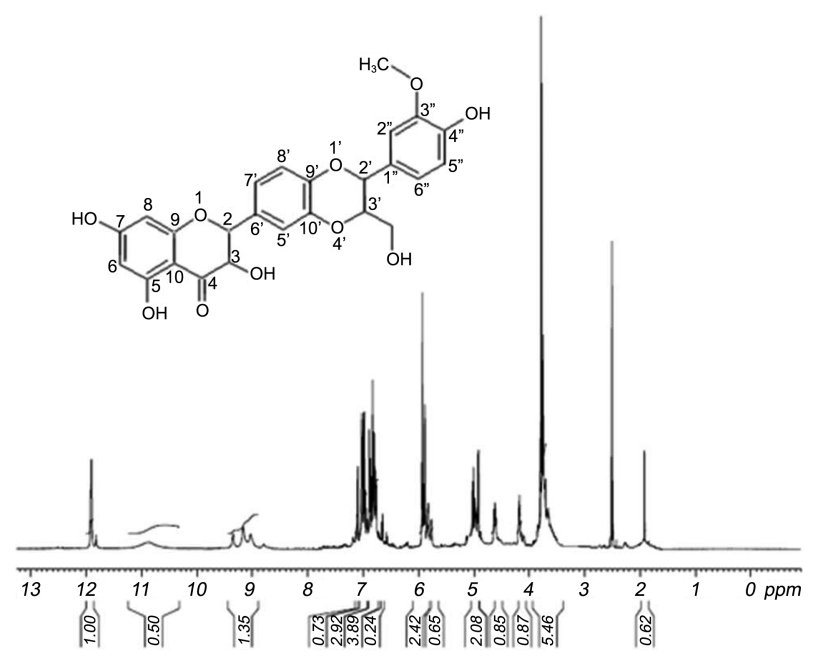

Figure 3. ${ }^{1} \mathrm{H}$ NMR spectrum of SIL.

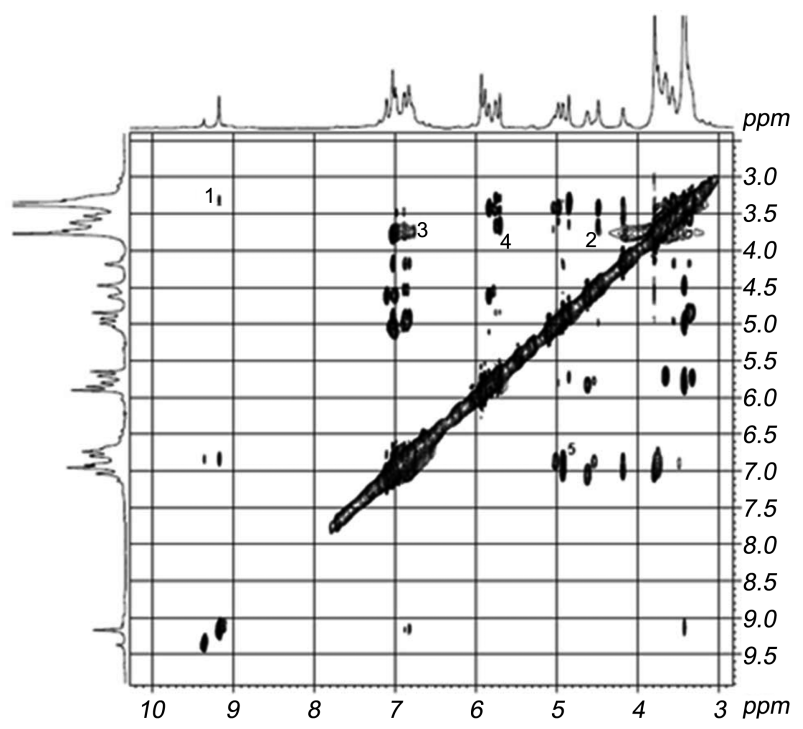

Figure 4. 2D ROESY NMR spectrum of SIL- $\beta-$ CD complex.

hydroxyl protons of $\beta-\mathrm{CD}$ (denoted as 2). The methylene protons in the benzodioxin ring of position $3^{\prime}$ correlate to the secondary hydroxyl groups in the wide rim of $\beta-\mathrm{CD}$ (denoted as 4). This correlation explains close proximity of secondary hydroxyl groups of $\beta-\mathrm{CD}$ to the methylene protons by encapsulation the substituted phenyl ring by the cavity of $\beta$ CD. The aromatic protons of methoxy substituted phenyl ring shows the off-diagonal peaks with $\mathrm{H} 1$ and $\mathrm{H} 2$ protons of $\beta$-CD (represented as 5 and 3 ).

Based on the above results, the structure of the host-guest complex may be represented schematically as shown in Figure 5.

Binding of SIL-BSA. The absorption spectra of BSA with varying concentrations of SIL are shown in Figure 6 (a). The concentration of BSA $\left(1 \times 10^{-5} \mathrm{~mol} \mathrm{dm}^{-3}\right)$ is unaltered and SIL is added in increasing concentrations. Initially there is a single absorption band observed in this titration corresponding to BSA at $277 \mathrm{~nm}$ and an additional absorption band is seen when SIL is added. The new band is due to 


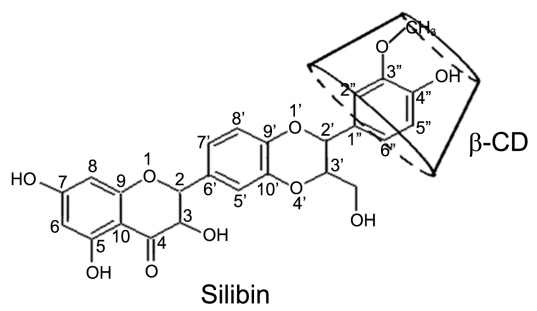

Figure 5. Schematic picture of the SIL- $\beta$-CD complex.
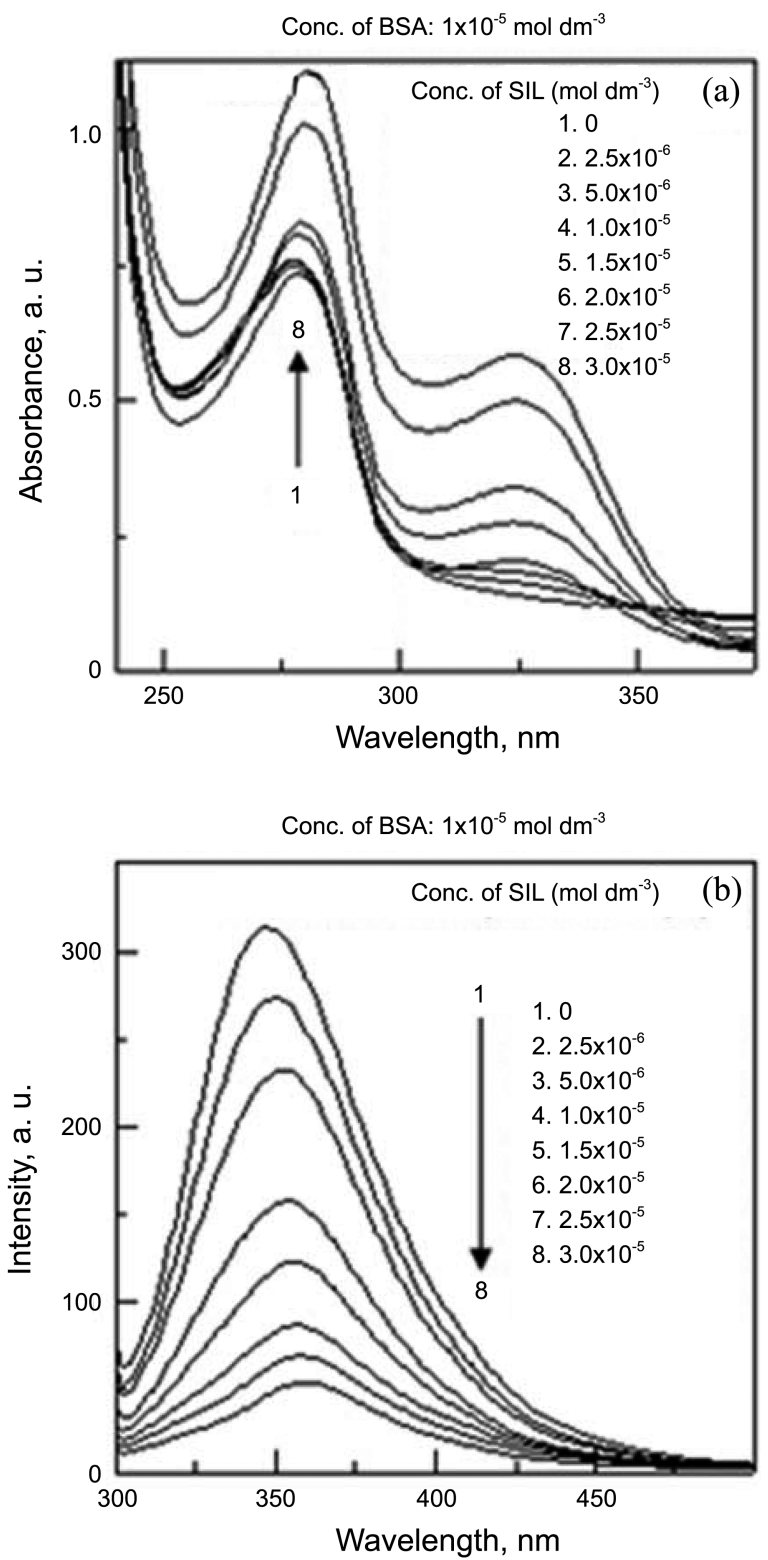

Figure 6. (a) Absorption spectra of BSA with varying concentrations of SIL (b) Fluorescence spectra of BSA with varying concentrations of SIL.

the absorption of SIL ( $325 \mathrm{~nm}$ ) and it corresponds to the highest occupied molecular orbital (HOMO) and the lowest unoccupied molecular orbital (LUMO) transition. These bands belong to the $\pi-\pi^{*}$ transition. The shift of band maxima indicates that there exists an interaction between SIL and BSA and a ground state association complex is formed.
Table 2. Absorption and fluorescence spectral data for the binding of SIL with BSA

\begin{tabular}{cccc}
\hline $\begin{array}{c}\text { Concentration of } \\
\text { SIL, mol dm }\end{array}$ & $\begin{array}{c}\text { Absorption } \\
\text { maxima, nm }\end{array}$ & $\begin{array}{c}\text { Absorbance, } \\
\text { a. u. }\end{array}$ & $\begin{array}{c}\text { Fluorescence } \\
\text { maxima, nm }\end{array}$ \\
\hline 0 & 277.0 & 0.754 & 347.0 \\
$2.5 \times 10^{-6}$ & 278.0 & 0.742 & 348.0 \\
& 325.0 & 0.161 & \\
$5.0 \times 10^{-6}$ & 278.0 & 0.754 & 348.0 \\
& 323.0 & 0.184 & \\
$1.0 \times 10^{-5}$ & 278.0 & 0.729 & 349.0 \\
& 324.0 & 0.202 & \\
$1.5 \times 10^{-5}$ & 279.0 & 0.801 & 349.0 \\
& 324.0 & 0.272 & \\
$2.0 \times 10^{-5}$ & 279.0 & 0.823 & 350.0 \\
& 324.0 & 0.337 & \\
$2.5 \times 10^{-5}$ & 280.0 & 1.002 & 350.0 \\
& 325.0 & 0.495 & \\
$3.0 \times 10^{-5}$ & 281.0 & 1.100 & 350.0 \\
& 325.0 & 0.579 &
\end{tabular}

Note: Excitation wavelength: $277 \mathrm{~nm}$

The fluorescence quenching of BSA with varying concentrations of SIL is shown in Figure 6(b). It illustrates that SIL quenched the fluorescence of BSA. The changes of absorbance and fluorescence spectral values on the addition of SIL to BSA are compiled in Table 2. The fluorescence quenching may be normally a result from a variety of processes such as energy transfer, ground state complex formation and collision processes. Collisional quenching is described by the popular Stern-Volmer equation (Eq. 6).

$$
\mathrm{F}_{0} / \mathrm{F}=1+\mathrm{K}_{\mathrm{sv}}[\mathrm{Q}]=\mathrm{k}_{\mathrm{q}} \tau_{0}[\mathrm{Q}]
$$

where $\mathrm{F}_{0}$ and $\mathrm{F}$ are the fluorescence intensities in the absence and the presence of quencher (SIL) respectively, $\mathrm{k}_{\mathrm{q}}$ is the bimolecular quenching constant, $\tau_{0}$ is the lifetime of the fluorophore in the absence of quencher, $[\mathrm{Q}]$ is the concentration of the quencher, and $\mathrm{K}_{\mathrm{SV}}=\mathrm{k}_{\mathrm{q}} \tau_{0}$ is the Stern-Volmer quenching constant. Figure 7(a) shows the Stern-Volmer plot of the quenching of BSA by SIL. The linearity of the plot for the SIL-BSA system suggests static quenching and SIL binds to BSA forming a complex with decreased fluorescence. The $\mathrm{K}_{\mathrm{Sv}}$ calculated for the binding is $1.76 \times 10^{5}$ $\mathrm{mol}^{-1} \mathrm{dm}^{3}$. The apparent binding constant $\mathrm{K}_{\mathrm{A}}$ and the number of binding sites for the binding of SIL with BSA can be determined from the following equation:

$$
\log _{10}\left(\frac{\mathrm{F}_{0}-\mathrm{F}}{\mathrm{F}}\right)=n \log _{10} \mathrm{~K}_{\mathrm{A}}-n \log _{10}\left(\frac{1}{\left[\mathrm{D}_{\mathrm{t}}\right]-\left(\mathrm{F}_{0}-\mathrm{F}\right)\left[\mathrm{P}_{\mathrm{t}}\right] / \mathrm{F}_{0}}\right)
$$

where $\mathrm{F}_{0}$ and $\mathrm{F}$ are the intensities of fluorescence before and after the addition of the quencher, $\left[\mathrm{D}_{t}\right]$ and $\left[\mathrm{P}_{t}\right]$ are the total concentration of SIL and BSA respectively. The plot of log $\left(\mathrm{F}_{0}-\mathrm{F}\right) / \mathrm{F} v$ s. $\log \left\{1 /\left[\mathrm{D}_{\mathrm{t}}\right]-\left(\mathrm{F}_{0}-\mathrm{F}\right)\left[\mathrm{P}_{\mathrm{t}}\right] / \mathrm{F}_{0}\right\}$ for the binding of SIL with BSA in water is shown in Figure 7(b). The binding constant $\mathrm{K}_{\mathrm{A}}$ for the SIL-BSA binding is determined as $1.4 \times$ 

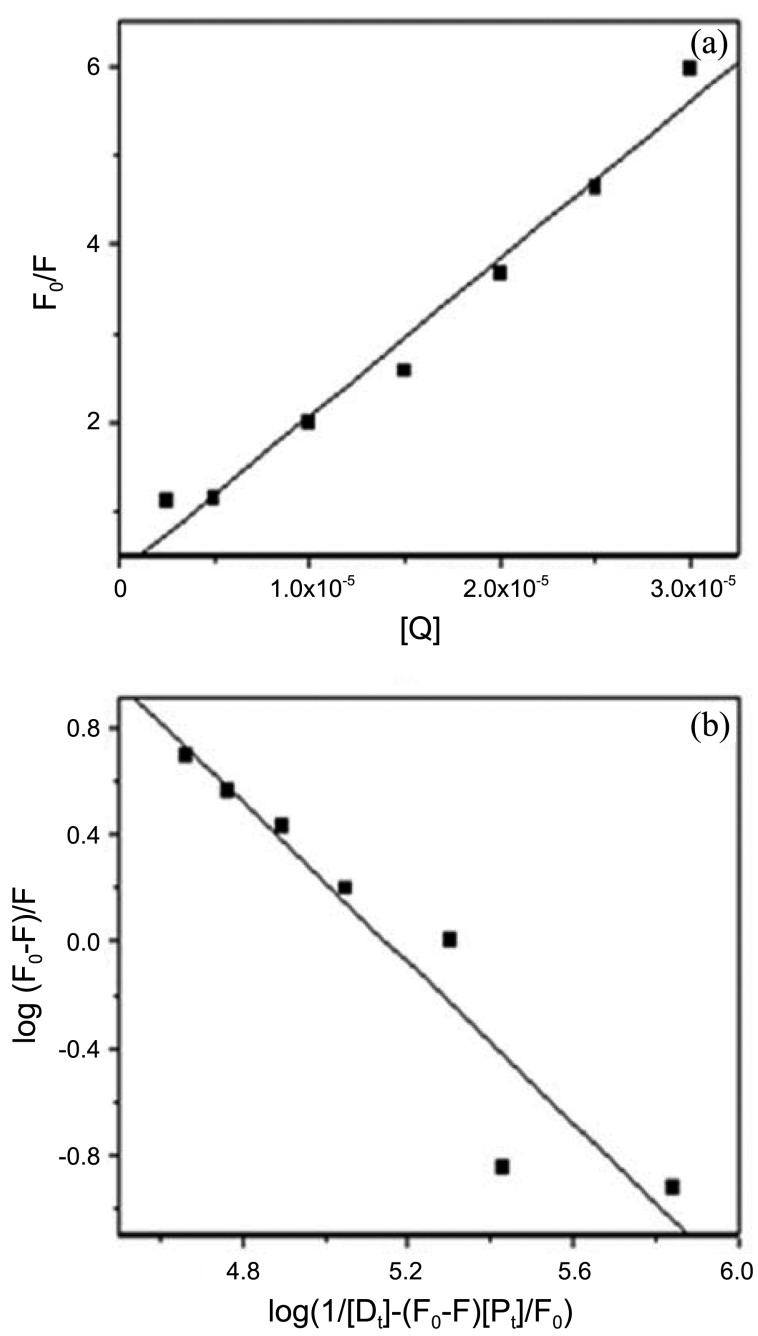

Figure 7. (a) Stern-Volmer plot for the quenching of BSA fluorescence by SIL (b) Plot of $\left(\mathrm{F}_{0}-\mathrm{F}\right) / \mathrm{F}$ vs. $\log \left\{1 /\left[\mathrm{D}_{\mathrm{t}}\right]-\left(\mathrm{F}_{0}-\mathrm{F}\right)\left[\mathrm{P}_{\mathrm{t}}\right] /\right.$ $\left.\mathrm{F}_{0}\right\}$ for the binding of SIL with BSA in water.

$10^{5} \mathrm{~mol}^{-1} \mathrm{dm}^{3}$ and the number of binding sites is 1.5 .

A 'spectroscopic ruler' for measuring the distance between two types of molecules interacting in a chemico-biological binding system is the Förster resonance energy transfer (FRET). It occurs when the fluorescence emission of the donor molecule overlaps with the excitation band of the acceptor molecule, both of them being within $8 \mathrm{~nm}$ distance of separation R. As it is seen in Figure 8 which displays the excitation spectrum of SIL with the fluorescence emission spectrum of BSA there is an area of overlap of the spectra. The distance $r$ between the SIL and BSA can be calculated using FRET following the Eq. (8),

$$
\mathrm{E}=1-\mathrm{F} / \mathrm{F}_{0}=\mathrm{R}^{6} /\left(\mathrm{R}^{6}+\mathrm{r}^{6}\right)
$$

where $r$ is the binding distance between the donor and the acceptor, $\mathrm{R}_{0}$ is the critical distance when the efficiency of excitation energy transferred to the acceptor is $50 \%$. Using the Förster formula the efficiency can be calculated.

$$
\mathrm{R}_{0}{ }^{6}=8.79 \times 10^{-25} \mathrm{~K}^{2} \mathrm{n}^{-4} \phi \mathrm{J}
$$

In this equation, $\mathrm{K}^{2}$ is the spatial orientation factor of the

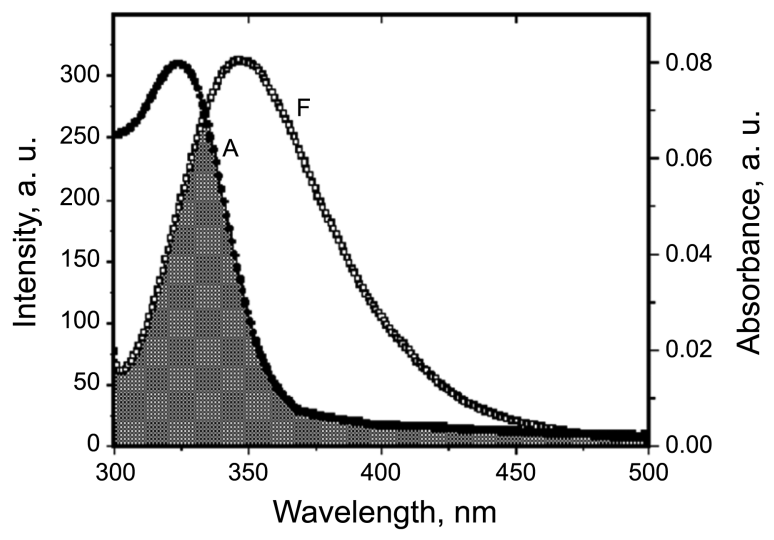

Figure 8. The excitation spectrum of SIL merged with the fluorescence emission spectrum of BSA showing the area of overlap.

dipole, $\mathrm{n}$ is the refractive index of the medium, $\phi$ is the fluorescence quantum yield of the donor, and $\mathrm{J}$ is the overlap integral of the fluorescence emission spectrum of the donor (BSA) with the absorption spectrum of the acceptor (SIL), which can be calculated by the following equation:

$$
\mathrm{J}=\frac{\int_{0}^{\infty} F_{\mathrm{D}}(\lambda) \varepsilon_{\mathrm{A}}\left(\lambda_{0}\right) \lambda^{4} \mathrm{~d} \lambda}{\int_{0}^{\infty} F_{\mathrm{D}}(\lambda) \mathrm{d} \lambda}
$$

where $F(\lambda)$ is the fluorescence intensity of the donor at wavelength $\lambda$ to $\lambda+\Delta \lambda, \varepsilon(\lambda)$ is the molar absorption coefficient of the acceptor at wavelength $\lambda$. Here, $\mathrm{K}^{2}=2 / 3, \mathrm{n}=$ 1.34 , and $\phi=0.15$. The calculated $\mathrm{J}$ is $4.53 \times 10^{-20} \mathrm{~cm}^{3}$ $\mathrm{mol}^{-1} \mathrm{dm}^{3}, \mathrm{R}_{0}$ is $3.22 \mathrm{~nm}, \mathrm{E}$ is 0.11 , and $\mathrm{r}$ is $4.58 \mathrm{~nm}$. The values for the Förster distance, $\mathrm{R}_{0}(=3.22 \mathrm{~nm})$ and $\mathrm{r}$ are below $8 \mathrm{~nm}$ and indicate a binding interaction between SIL and BSA.

Binding of SIL- $\beta-C D$ Complex with BSA. The SIL molecule is capable of binding to $\beta$-CD with a significant binding strength, as discussed in the previous section. Hence, in order to understand the role of $\beta-\mathrm{CD}$ in the interaction of SIL with BSA, absorption and fluorescence spectra are recorded for the binding titration of SIL- $\beta$-CD complex with BSA. Figure 9(a) shows the absorption spectra of BSA

Table 3. Absorption and fluorescence spectral data for the binding of SIL $-\beta-C D$ with BSA

\begin{tabular}{cccc}
\hline $\begin{array}{c}\text { Concentration of } \\
\text { SIL, mol dm }\end{array}$ & $\begin{array}{c}\text { Absorption } \\
\text { maxima, nm }\end{array}$ & $\begin{array}{c}\text { Absorbance, } \\
\text { a. u. }\end{array}$ & $\begin{array}{c}\text { Fluorescence } \\
\text { maxima, nm }\end{array}$ \\
\hline 0 & 277.0 & 1.229 & 347.0 \\
$2.5 \times 10^{-6}$ & 277.0 & 1.267 & 350.0 \\
$1.0 \times 10^{-5}$ & 277.0 & 1.299 & 353.0 \\
$1.5 \times 10^{-5}$ & 277.0 & 1.312 & 354.0 \\
$2.0 \times 10^{-5}$ & 278.0 & 1.294 & 355.0 \\
$2.5 \times 10^{-5}$ & 280.0 & 1.476 & 357.0 \\
& 323.0 & 0.599 & \\
$3.0 \times 10^{-5}$ & 279.0 & 1.447 & 357.0 \\
& 321.0 & 0.607 & \\
\hline
\end{tabular}

Note: Excitation wavelength: $277 \mathrm{~nm}$ 

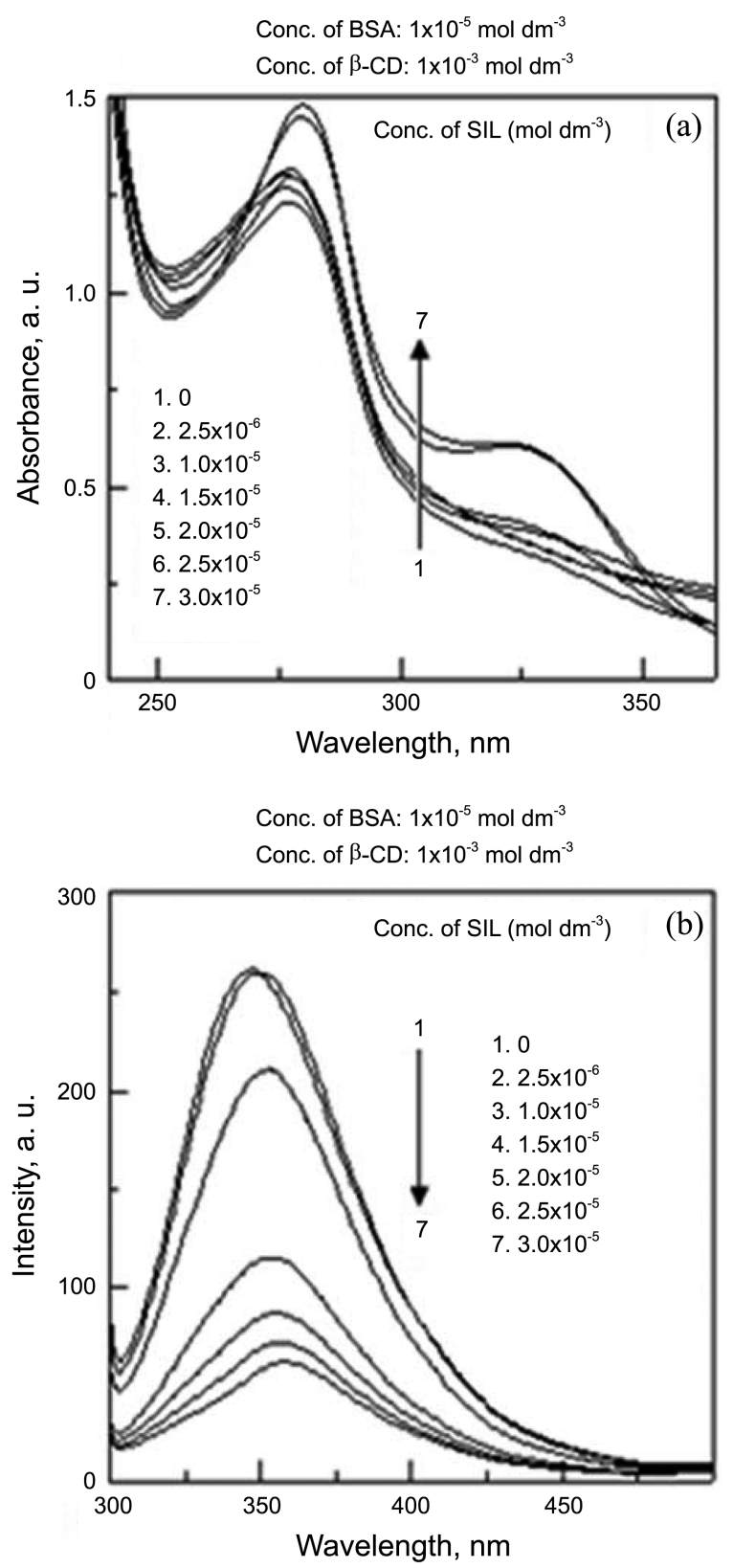

Figure 9. (a) Absorption spectra of BSA with varying concentrations of SIL- $\beta$-CD (b) Fluorescence spectra of BSA with varying concentrations of SIL $-\beta-\mathrm{CD}$.

without and with various amounts of the added SIL $-\beta-C D$. The absorbance is found enhanced with each addition, due to the interaction of the $\beta$-CD-bound SIL with BSA. The absorption and fluorescence spectral data are compiled in Table 3. The fluorescence spectra of the tryptophan fluorescence of BSA with varied amounts of SIL $-\beta-C D$ are shown in Figure 9(b).

The fluorescence shows a quenching and the SternVolmer plot for this quenching is linear as shown in Figure 10 (a). The $\mathrm{K}_{\mathrm{Sv}}$ for the quenching is calculated as $1.25 \times 10^{5}$ $\mathrm{mol}^{-1} \mathrm{dm}^{3}$. This value is smaller than the $\mathrm{K}_{\mathrm{SV}}$ for the binding interaction of free SIL with BSA. Figure 10(b) shows the plot of $\log \left(\mathrm{F}_{0}-\mathrm{F}\right) / \mathrm{F} v s . \log \left\{1 /\left[\mathrm{D}_{\mathrm{t}}\right]-\left(\mathrm{F}_{0}-\mathrm{F}\right)\left[\mathrm{P}_{\mathrm{t}}\right] / \mathrm{F}_{0}\right\}$ for the binding of SIL with BSA in the presence of $\beta$-CD. From the
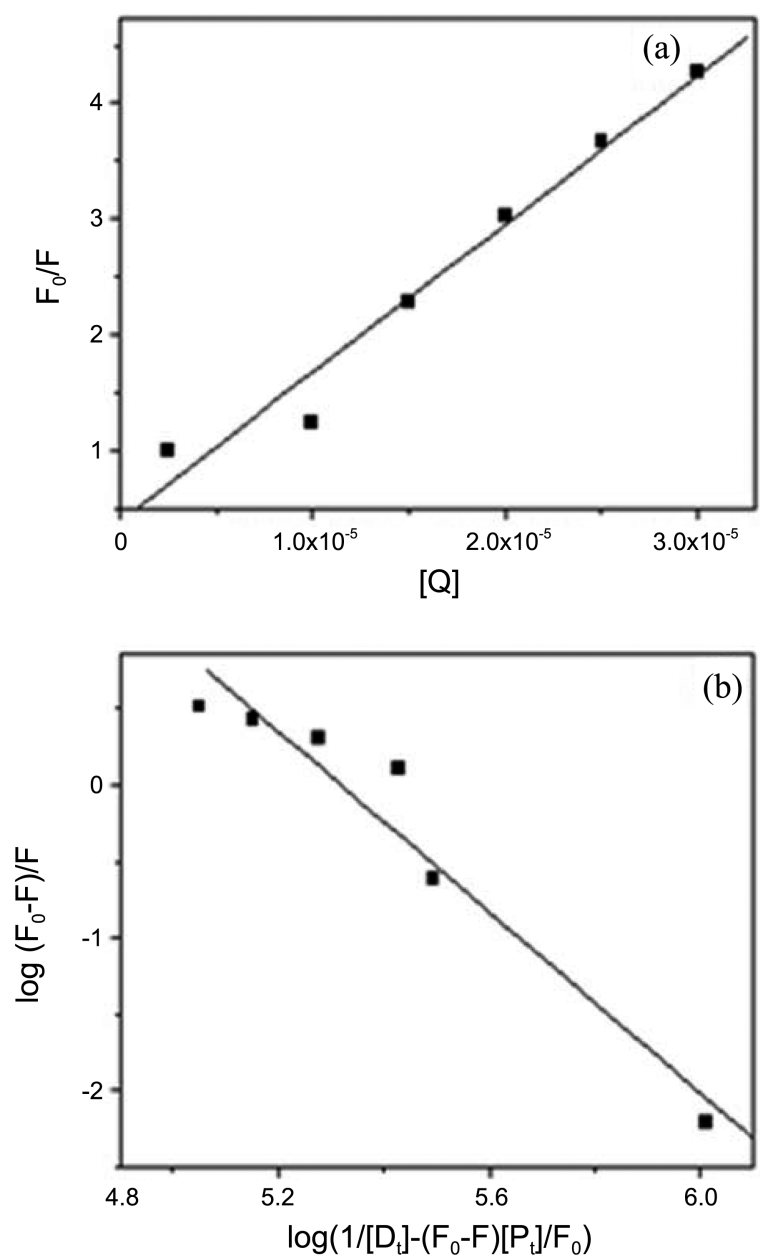

Figure 10. (a) Stern-Volmer plot for the quenching of BSA fluorescence by SIL- $\beta$-CD (b) Plot of $\left(\mathrm{F}_{0}-\mathrm{F}\right) / \mathrm{F}$ vs. $\log \left\{1 /\left[\mathrm{D}_{\mathrm{t}}\right]-\left(\mathrm{F}_{0}-\right.\right.$ $\left.\mathrm{F})\left[\mathrm{P}_{\mathrm{t}}\right] / \mathrm{F}_{0}\right\}$ for the binding of SIL with BSA in $\beta$-CD.

linear plot the binding constant $\left(\mathrm{K}_{\mathrm{A}}\right)$ is calculated as $8.31 \times$ $10^{4} \mathrm{dm}^{3} \mathrm{~mol}^{-1}$ and the binding site is 2.95 . This binding constant is quite smaller than that observed in the case of free SIL-BSA binding in the absence of $\beta$-CD. This suggests that the $\beta$-CD molecule encapsulates the SIL molecule and blocks it from direct collision with Trp residues in the binding pocket of BSA.

The FRET calculation for SIL- $\beta$-CD/BSA binding in a similar method as that described for SIL-BSA binding results in the following data: $\mathrm{J}=8.30 \times 10^{-20} \mathrm{~cm}^{3} \mathrm{~mol}^{-1} \mathrm{dm}^{3}$, $\mathrm{R}_{0}=3.56 \mathrm{~nm}, \mathrm{r}=8.29 \mathrm{~nm}, \mathrm{E}=0.0062$. The Förster distance $(=3.56 \mathrm{~nm})$ is within $8 \mathrm{~nm}$. However, the distance is slightly higher than that observed in the free SIL bound to BSA. These results reveal that the FRET efficiency is decreased in the case of the $\beta$-CD-bound SIL interacting with BSA owing to the encapsulation and hence the restricted environment supplied by $\beta-C D$. Figure 11 shows the overlap of the excitation spectrum of SIL- $\beta$-CD with the fluorescence emission spectrum of BSA. Hence clearly $\beta$-CD modulates the binding interaction of SIL with BSA.

Molecular Modeling of SIL-BSA Binding. Molecular docking technique is used to extend further insight into the 


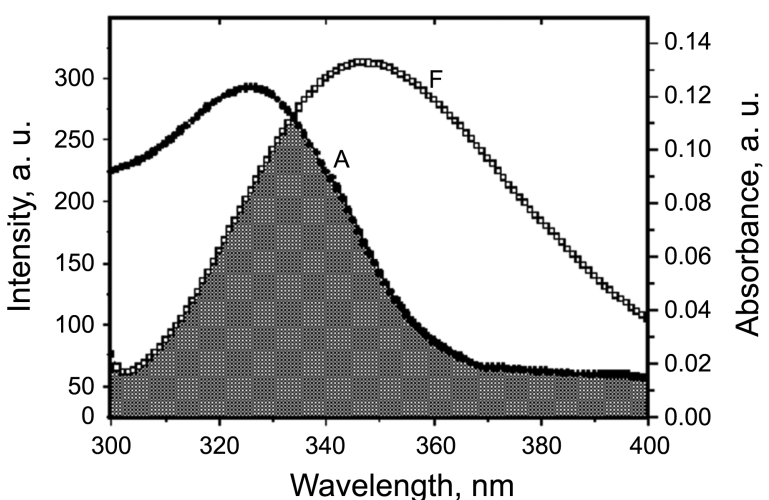

Figure 11. The overlap of the excitation spectrum of SIL- $\beta-C D$ with the fluorescence emission spectrum of BSA.

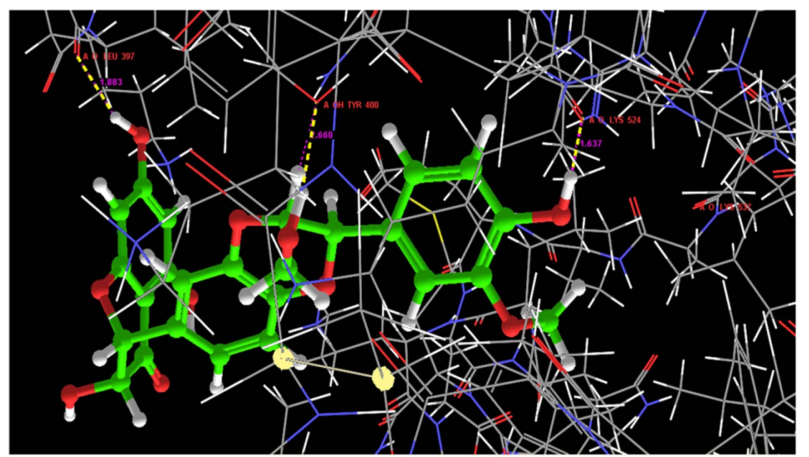

Figure 12. Molecular docking poses of hydrogen bonding interaction between SIL and BSA.

mode of SIL-BSA binding. The structures of the molecules were refined by assigning the bonds, bond orders, charge and hybridization, creating explicit hydrogen and this was repeated. BSA consists of three homologous domains (I, II, and III): I (residues 1-183), II (184-376), III (377-583), each containing two sub-domains (A and B) that assemble to make it a heart shaped molecule. There is a large hydrophobic cavity in sub-domain IIA to accommodate the drug molecule, which plays an important role in the transportation of drugs in BSA. The best energy ranked results (Fig. 12) reveal that SIL is located within the sub-domain III hydrophobic cavity in close proximity to the residues, such as Lys524, Leu-397, and Tyr-400 suggesting the existence of hydrophobic interaction between them.

Moreover, clearly hydrogen bonding interactions are revealed at these sites. Hence, this finding provides a good structural basis to explain the efficient quenching of fluorescence of BSA by SIL. Furthermore, there are also a number of hydrophobic interactions, because several apolar residues in the proximity of the ligand play an important role in stabilizing the molecule via phobic interactions. As shown in the figure the hydrogen bonding occurs, due to the presence of the hydroxyls of the SIL molecule, with the Lys-524, Leu397 , and Tyr-400 residues of BSA. These hydrogen bonds increase the stability of the SIL-BSA bound system. Therefore, the interaction between the SIL and BSA is dominated by hydrogen bonds along with hydrophobic interaction. Since
$\beta$-CD blocks some of the possible hydrogen bond formation between SIL and BSA, the binding strength is relatively low compared to that in water.

\section{Conclusions}

In this work, we studied the interaction of Silybin in free form and $\beta$-CD-bound form with bovine serum albumin by fluorescence spectroscopy and UV-visible absorption spectroscopy. The stoichiometry of the inclusion complex of SIL with $\beta-\mathrm{CD}$ is $1: 1$ and the mode of binding is that the methoxy substituted phenyl ring is inside the cyclodextrin cavity. There is an isosbestic point in the absorption spectra of SIL at various $\mathrm{pH}$ suggesting equilibrium between a neutral and cationic form of SIL as there are hydroxyl groups in the aromatic rings which are possible deprotonated. This is not clearly observed for the same equilibrium in the presence of $\beta$-CD which suggests that the encapsulation by $\beta$-CD offers restriction for the access of SIL by protons. The ground state $\mathrm{p} K_{\mathrm{a}}$ for the neutral-cation equilibrium of SIL is calculated using the relations described above as 5.24. The structure of the inclusion complex is proposed using the results from fluorescence and NMR spectroscopic results. Quenching of BSA fluorescence by SIL occurs as a result of the formation of SIL-BSA complex. Molecular docking studies confirm the interaction between the SIL and BSA. In the presence of $\beta-C D$, the $K_{S v}$ and the binding constant values of the quenching due to the binding of SIL with BSA is decreased which suggests the blocking of the hydrogen bonding and phobic interaction of SIL with BSA. Hence $\beta$-CD modulates the binding of SIL with BSA.

Conflict of Interest. The authors declare no conflict of interest.

Acknowledgments. Publication cost of this paper was supported by the Korean Chemical Society.

\section{References}

1. Turnidge, J. Drugs 1999, 58, 29-36.

2. Sinjan, C.; Nand, K. Chem. Biol. Drug Des. 2012, 80, 693-705.

3. Maliwal, B. P.; Rao, A. G. A.; Rao, M. S. N. Int. J. Pept. Protein Res. 1985, 25, 382-388.

4. Meier-Kriesche, H. U.; Shaw, L. M. Korecka, M.; Kaplan, B. Ther. Drug Monit. 2000, 22, 27-30.

5. Kessler, K. F.; Barth, R. F.; Wong, K. P. Int. J. Pept. Protein Res. 1982, 20, 73-80.

6. Liu, J.; Begley, D.; Mitchell, D. D.; Verlinde, C. L. M. J.; Varani, G.; Fan, E. Chem. Biol. Drug Des. 2008, 71, 408-419.

7. Dutta, S. K.; Basu, S. K.; Sen, K. K. Indian J. Exp. Biol. 2006, 44, 123-127.

8. Carter, D. C.; Ho, J. X. Adv. Protein Chem. 1994, 45, 153-203.

9. Ayranci, E.; Duman, O. Food Chem. 2004, 84, 539-543.

10. Qu, S. S.; Liu, Y.; Wang, T. Z.; Gao, W. Y. Chemosphere 2002, 4, 1211-1214.

11. Jhy-Wen, W.; Lie-Chwen, L.; Shih-Chieh, H.; Chi-Hung, L.; ChinWen, C.; Tung-Hu, T. Drug Metab. Dispos. 2008, 36, 589-596.

12. Katiyar, S. K. Int. J. Oncol. 2005, 26, 169-176.

13. Katiyar, S. K.; Roy, A. M.; Baliga, M. S. Mol. Cancer Ther. 2005, 
4, 207-216.

14. Gažák, R.; Svobodová, A.; Psotová, J.; Sedmera, P.; Přikrylová, V.; Walterová, D.; Křen, V. Bioorg. Med. Chem. 2004, 12, 56775687.

15. Pelt, J. V.; Verslype, C.; Crabbé, T.; Zaman, Z.; Fevery, J. Alcohol Alcsm. 2003, 38, 411-414.

16. Křen, V.; Walterováb, D. Biomed. Papers 2005, 149, 29-41.

17. Singh, R. P.; Agarwal, R. Eur. J. Cancer 2005, 41, 1969-1979.

18. Rice-Evans, C. A.; Miller, N. J.; Paganga, G. Free Radic. Biol. Med. 1996, 20, 933-956.

19. Heim, K. E.; Tagliaferro, A. R.; Bobilya, D. J. J. Nutr. Biochem. 2002, 13, 572-584.

20. Gyorgy, I.; Antus, S.; Foldiak, G. Radiat. Phys. Chem. 1992, 39,
81-84.

21. Enoch, I. V. M. V.; Rajamohan, R.; Swaminathan, M. Spectrochim. Acta A 2010, 77, 473-477.

22. Enoch, I. V. M. V.; Sameena, Y. J. Solution Chem. 2013, 42, 470484.

23. Sudha, N.; Enoch, I. V. M. V. J. Solution Chem. 2011, 40, 17551768.

24. Benesi, H. A.; Hildebrand, J. H. J. Am. Chem. Soc. 1949, 71, 2703-2707.

25. Arnold, B.; Euler, A.; Fields, K.; Zaini, R. J. Phys. Org. Chem. 2000, 13, 729-734.

26. Jorgenson, M.; Hartter, D. R. J. Am. Chem. Soc. 1963, 85, 878883 . 